\title{
Determinants of Increased Left Ventricular Output during In Utero Ventilation in Fetal Sheep
}

\author{
R. M. LEWINSKY, R. S. SZWARC, L. N. BENSON, AND J. W. K. RITCHIE \\ Division of Perinatology, The Samuel Lunenfeld Research Institute, Mount Sinai Hospital \\ [R.M.L., J.W.K.R.], and The Division of Pediatric Cardiology, The Hospital for Sick Children \\ [R.S.S., L.N.B.], University of Toronto, Toronto, Ontario M5G 1X5, Canada
}

\begin{abstract}
A model of in utero ventilation was used to elucidate the mechanisms by which left ventricular (LV) output increases with the transition from a fetal to a neonatal circulation. Using a conductance catheter, LV volumes were measured in seven anesthetized, near-term fetal sheep. Pressure-volume data was recorded before and with oxygen ventilation and again after occlusion of the umbilical cord. Ventilation caused increases in LV end-diastolic volume $(2.3 \pm 0.9$ to $2.9 \pm 0.6 \mathrm{~mL} / \mathrm{kg} ; p<0.05)$, stroke volume $(1.2 \pm 0.3$ to $1.9 \pm 0.2 \mathrm{~mL} / \mathrm{kg} ; p<0.001)$, and ejection fraction $(52.8 \pm 11.1$ to $66.4 \pm 8.8 \% ; p<0.001)$. Contractile state, as assessed by end-systolic elastance, did not change during the transition. Heart rate also remained constant. Afterload, as assessed by effective arterial elastance, decreased from $1.80 \pm 0.37$ to $1.04 \pm 0.33$ $\mathrm{kPa} / \mathrm{mL}(p<0.01)$. Occlusion of the umbilical cord did not result in any further change in hemodynamic parameters.
\end{abstract}

ABSTRACT

Pressure-volume analysis revealed that a decrease in effective LV afterload and an increased LV end-diastolic volume are the major determinants of, and contribute comparably to, the profound increase in LV output with in utero ventilation. Enhanced contractility is not required for the increase in LV output to occur. (Pediatr Res 36: 373-379, 1994)

LV, left ventricle

\section{Abbreviations}

RV, right ventricle

$\mathbf{d P} / \mathbf{d t}_{\max }$, the maximum of the first time derivative of the LV pressure wave form

$\mathbf{E}_{\text {es }}$, end-systolic elastance

$\mathbf{E}_{\text {aLV }}$, effective arterial elastance (afterload opposing LV ejection)

PV, pressure-volume
Adaptation of the fetus to extrauterine life involves a number of rapidly occurring processes. Among these, the initiation of breathing and the transition from a fetal to a neonatal circulation are the paramount events. In the fetal heart, which is characterized by RV dominance (1-3), the ventricles operate in parallel. After birth, the ventricles function serially and LV output increases 2- to 3-fold (4-10). The mechanism by which the LV increases its output to such an extent remains a mystery.

In utero ventilation is associated with a marked increase in LV output identical with the increase observed in the immediate postpartum state. Either an increase in end-diastolic volume, ejection fraction, or both may underlie the augmented LV stroke volume. Ejection fraction could increase due to an increase in contractility or a reduction in afterload. Augmented LV end-diastolic

Received March 22, 1993; accepted March 27, 1994.

Correspondence and reprint requests: R. M. Lewinsky, M.D., Department of Perinatology, Mount Sinai Hospital, Room 775, 6000 University Ave., Toronto, Ontario M5G 1X5, Canada.

Supported by a grant from The Medical Research Council of Canada. volume as a result of increased pulmonary venous return (11-15) and an increase in contractility $(16,17)$ have both been suggested to play a role in augmenting LV output at birth. Because systemic pressure is increased or unchanged at birth, it has been suggested that a decrease in afterload is unlikely to occur (18). On the other hand, afterload may decrease due to the exposure of the LV to the pulmonary circulation through the patent ductus arteriosus (19). The individual contribution of each component to the augmentation of LV performance at birth is unknown.

We applied the conductance catheter technique to the in utero ventilation model. This technique, which provides continuous and instantaneous measurements of ventricular volume, allowed changes in LV end-diastolic volume, stroke volume, and ejection fraction to be determined. Contractility was assessed by $\mathrm{E}_{\mathrm{es}}$, a relatively load- and heart rate-independent index $(20,21)$, and the $E_{a L V}$ was used to evaluate afterload $(22,23)$. This study furthers our understanding of the mechanisms by which the LV increases its output during the transition from a fetal to a neonatal circulation. 


\section{METHODS}

Surgical procedure. This study was approved by the Mount Sinai Hospital Animal Care Committee following the guidelines of the Canadian Council for Animal Care. Surgery was performed on seven pregnant ewes between 138 and $141 \mathrm{~d}$ of gestation. Anesthesia was induced with i.v. thiopental sodium and maintained by positive pressure ventilation with $1-1.5 \%$ isoflurane and oxygen. A vascular catheter was placed in the femoral artery of the ewe to monitor maternal blood pressure and gases. The upper part of the fetus was then withdrawn through a uterine incision up to the insertion of the umbilical cord. A catheter was placed in an axillary artery to obtain fetal blood gases. An inflatable cuff occluder was then sewn around the umbilical cord. The occluder was constructed from the balloon of a Foley catheter (75-mL capacity) tethered inside a Fiberglas screen cuff. During experiments, the cuff was inflated with $60 \mathrm{~mL}$ of air to gently occlude the umbilical cord over a $6-\mathrm{cm}$ length. The function of the occluder was confirmed at necropsy. A $2 \mathrm{~F}$ catheter-tip pressure transducer (Millar, Houston, TX) was introduced through a left carotid artery cutdown, advanced into the LV, and positioned such that no impact artifact was evident. A custom-made, $4 \mathrm{~F}$, eightelectrode conductance catheter was similarly advanced through the same carotid artery and positioned in the LV such that its distal electrode was at the apex and the most proximal electrode cephalad of the aortic valve. Correct placement of the conductance catheter in the LV was determined by monitoring the segmental volume phase relationships and counterclockwise PV loop formation. The conductance catheter total electrode spacing was 4 $\mathrm{cm}$, the appropriate length being established from LV long axis measurements previously made on fetuses of the same gestational age. Correct catheter position was confirmed at necropsy. A venous line was advanced through the left jugular vein to the right atrium. A tracheostomy tube (5-mm inner diameter) was placed in the trachea through a midline neck incision, and a T-connector (4-mm inner diameter) was used to attach it to the inflow and outflow tubes (5-mm inner diameter) with the junction made at the trachea to minimize dead space. The fetus was then replaced and the uterine incision closed.

Experimental protocol. Hemodynamic recordings were acquired during three phases of the experiment: 1 ) during a control period, 2 ) during ventilation with $100 \%$ oxygen and, 3) during oxygen ventilation after umbilical cord occlusion. Before each set of hemodynamic recordings, fetal arterial blood samples $(0.3 \mathrm{~mL})$ were obtained and immediately analyzed for gas tensions and $\mathrm{pH}$ using a blood gas analyzer (model 170, Corning Medical, Medfield, MA) and temperature corrected to $39^{\circ} \mathrm{C}$. At each phase, steady state, instantaneous LV PV data were digitally recorded with both maternal and fetal ventilators off at end-expiration.

The experimental protocol began with control fetal recordings taken after surgery, when hemodynamic pa- rameters were observed to be stable. Tracheal fluid was then allowed to drain by gravity, and the inflow and outflow tubes were connected to a volume-regulated ventilator (model LS104-150, Bourns, Riverside, CA). To cause a maximal decrease in pulmonary vascular resistance, the fetuses were ventilated with $100 \%$ oxygen, with ventilator settings adjusted to deliver a tidal volume of about $10 \mathrm{~mL} / \mathrm{kg}$ at a rate of $30-50$ breaths $/ \mathrm{min}$ with peak inspiratory pressures not exceeding $40 \mathrm{~cm} \mathrm{H}_{2} \mathrm{O}$. A positive end-expiratory pressure of $5-8 \mathrm{~cm} \mathrm{H}_{2} \mathrm{O}$ was applied to offset amniotic pressure. Arterial blood gases and oxygen saturations were measured, and the ventilator settings were adjusted until acceptable values were reached $\left(\mathrm{Hb} \mathrm{O}_{2}\right.$ saturation $\geq 90 \%$, a decrease in $\mathrm{PCO}_{2} \geq$ $1.33 \mathrm{kPa}$ ). Ventilation was maintained for at least $15 \mathrm{~min}$, after which hemodynamic recordings were taken. The umbilical cord occluder was then inflated, with PV data being acquired simultaneously. Hemodynamic recordings were taken again 20 min after cord occlusion.

Hemodynamic data acquisition and analysis. The principles, technique, and validation of the conductance catheter method of $L V$ volume estimation are described in detail elsewhere (24-30). Briefly, the conductance catheter was connected to a Z-cath signal conditioner (IVM Systems Inc., Willowdale, Ontario, Canada) that applied an excitation current to the outer electrodes and measured the five segmental conductances $\left(G_{i}\right)$ between intervening electrode pairs. The instantaneous $L V$ volume was then obtained by:

$$
V(t)=\frac{L^{2} \cdot \rho}{\alpha}\left(\sum_{i=1}^{5} G_{i}(t)+\frac{1}{3} G_{1}(t)\right)-V_{c}
$$

where $\alpha$ is a unitless, empirical coefficient relating conductance catheter-derived stroke volume to actual stroke volume; $\alpha$ was assumed to be 1 in this study. $L$ is the interelectrode spacing, and $\rho$ is the specific electrical resistance of blood that was measured with a $0.5-\mathrm{mL}$ cuvette at each phase of the experiment. The parallel conductance volume, $\mathrm{V}_{c}$, is a correction term required to account for the conductivity of structures surrounding the LV blood pool. It was determined by a dilution method (26), whereby the conductivity of blood was transiently altered by a bolus injection of $0.3 \mathrm{~mL}$ of $3 \mathrm{M}$ $\mathrm{NaCl}$ into the right atrium. A portion of the saline immediately shunted through the foramen ovale and entered the $L V$, the remainder entering the RV. The saline entering the RV may have affected parallel conductance, thus rendering the first LV wash-in curve unreliable. Of the saline that entered the $R V$, a portion passed through the pulmonary circulation, which resulted in a second LV wash-in curve (Fig. 1). By the time the second curve was observed, most of the saline would have washed out of the RV. Thus, the second response was used to compute $V_{c}$ and was the mean value obtained from three consecutive saline injections. Left ventricular pressure, five segmental conductances, and ECG were digitized (250 


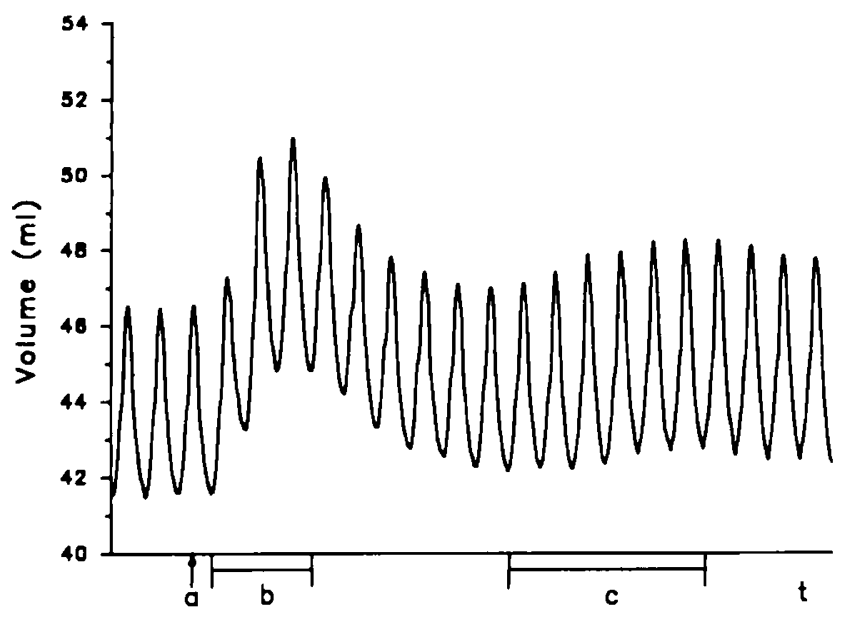

Figure 1. Example of the dual saline wash-in response of the fetal LV. Time of injection of hypertonic saline is indicated by the arrow $(a)$. The first transient increase in the signal $(b)$ is a result of saline shunted through the foramen ovale. The second transient increase $(c)$, which is used for calculation of parallel conductance volume, is a result of saline washing into the LV after passing through the lungs.

$\mathrm{Hz}, 12$ bit) on an IBM AT compatible computer and stored on hard disk for subsequent analysis.

Left ventricular stroke volume and heart rate were measured and LV output and $\mathrm{dP} / \mathrm{dt}_{\max }$ computed during each phase of the experiment. Contractility of the LV was assessed by $E_{e s}$, which was computed by single-beat analysis of the PV loop under steady state conditions using the "cosine" method $(24,31)$ :

$$
\mathrm{E}_{\mathrm{es}}=\frac{\left(\frac{\frac{\mathrm{dP}}{\mathrm{dt}_{\max }} \cdot T}{2 \pi}+\mathrm{P}_{\mathrm{DC}}\right)-\mathrm{P}_{\mathrm{es}}}{\mathrm{V}_{\mathrm{ed}}-\mathrm{V}_{\mathrm{es}}}
$$

where $\mathrm{T}$ is the period of an extrapolated isovolumic contraction and is estimated as twice the interval from time of $\mathrm{dP} / \mathrm{dt}_{\max }$ to $\mathrm{dP} / \mathrm{dt}_{\min }, \mathrm{P}_{\mathrm{DC}}$ is the pressure at which $\mathrm{dP} / \mathrm{dt}_{\max }$ occurs, $V_{e s}$ and $P_{e s}$ are the volume and pressure at endsystole, which was defined as the time when the maximum ratio of $L V$ pressure to volume occurs, and $V_{e d}$ was defined as the maximum volume. Afterload opposing left ventricular ejection was assessed by measuring $E_{a L v}$, the ratio of LV end-systolic pressure to stroke volume.

PV loop analysis was used to estimate the relative contributions of changes in end-diastolic volume $\left(V_{e d}\right)$ and $\mathrm{E}_{\mathrm{aLV}}$ to changes in stroke volume (SV) (Fig. 2). First, the SV after ventilation was predicted assuming an isolated increase in $\mathrm{V}_{\mathrm{ed}}$ by:

$$
\mathrm{SV}=\mathrm{V}_{\mathrm{ed}} \cdot \mathrm{EF}_{\mathrm{BL}}
$$

where $\mathrm{EF}_{\mathrm{BL}}$ is the baseline ejection fraction. The impact of reduced $E_{a L v}$ on $S V$ was then determined by assuming no change in $E_{e s}$ or $V_{e d}$. $E_{e s}$ and $E_{a L V}$ can be expressed as:

$$
E_{e s}=\frac{P_{e s}}{V_{e s}-V_{0}}
$$

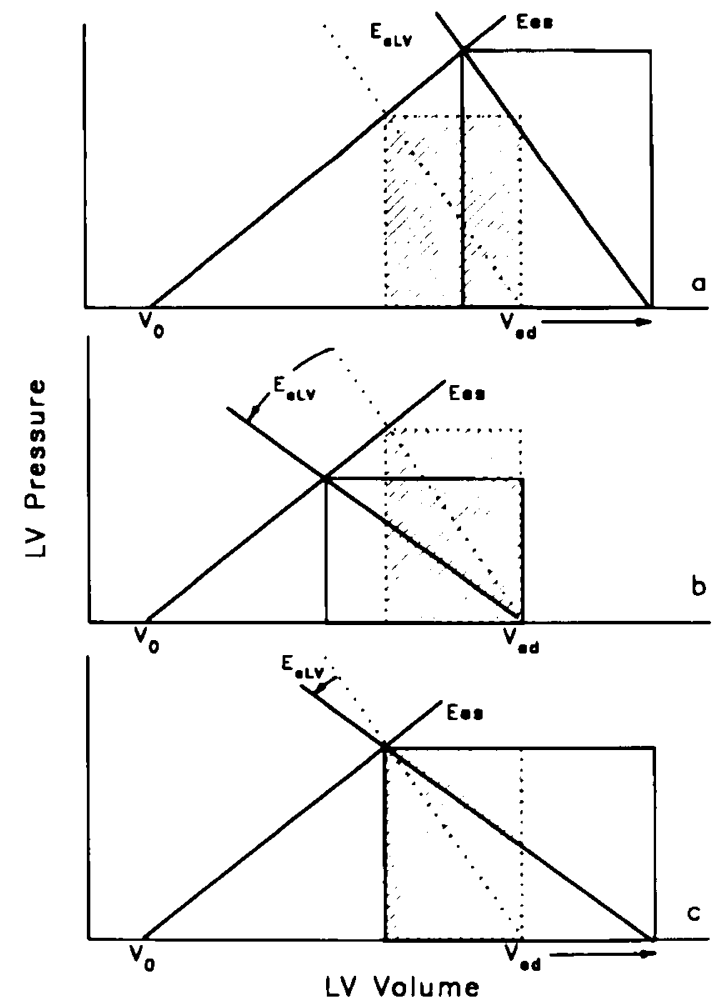

Figure 2. Schematic diagram using the PV plane to illustrate the effects of reduced effective $L V$ afterload $\left(E_{a L V}\right)$ and increased end-diastolic volume $\left(V_{e d}\right)$ on $L V$ stroke volume when contractility, measured by $\mathrm{E}_{\mathrm{es}}$, remains constant. The hatched rectangles represent control PV loops. An isolated increase in $\mathrm{V}_{\mathrm{es}}$, with $\mathrm{E}_{\mathrm{aL}, \mathrm{V}}$ unchanged, would result in an increased stroke volume, generating a higher systolic pressure $(a)$. An isolated reduction in $E_{a t . V}, V_{e d}$ unchanged, would also result in an increased stroke volume at a lower systolic pressure $(b)$. When decreased $E_{a L v}$ is combined with an increased $V_{c d}$, the effects on stroke volume and systolic pressure are additive $(c)$.

$$
E_{a L V}=\frac{P_{e s}}{V_{e s}-V_{e d}}
$$

where $V_{e s}$ and $P_{e s}$ are end-systolic volume and pressure and $V_{0}$ is the volume axis intercept of the $E_{e s}$ line. Equations 2 and 3 were then rearranged and equated to give an expression for $\mathrm{V}_{\mathrm{es}}$ :

$$
V_{e s}=\frac{E_{e s} \cdot \mathrm{V}_{0}-\mathrm{E}_{\mathrm{aLV}} \cdot \mathrm{V}_{\mathrm{ed}}}{\mathrm{E}_{\mathrm{es}}-\mathrm{E}_{\mathrm{aLV}}}
$$

Substitution for $\mathrm{V}_{0}$ from equation 2 allows prediction of SV by:

$$
S V=V_{e d}-\frac{E_{e s} \cdot \mathrm{V}_{\mathrm{es}}-\mathrm{E}_{\mathrm{aLV}} \cdot \mathrm{V}_{\mathrm{ed}}-\mathrm{P}_{\mathrm{es}}}{\mathrm{E}_{\mathrm{es}}-\mathrm{E}_{\mathrm{aLV}}}
$$

The increases in SV predicted by equations 1 and 5 were then expressed as a percentage of the observed increase by:

$$
\frac{S V_{\text {predicted }}-\mathrm{SV}_{\text {baseline }}}{S V_{\text {observed }}-S V_{\text {baseline }}} \times 100
$$

Statistical analysis. All variables and derived parameters were calculated from an average of 20 consecutive 
cardiac cycle measurements. A paired $t$ test was used to compare control data with values obtained during ventilation and ventilation data with values obtained after cord occlusion. Significance was assumed at the $p<0.05$ level. For control and ventilation periods, data are presented from seven animals. One animal was excluded from the cord occlusion stage due to technical difficulties. All data are expressed as mean $\pm 1 \mathrm{SD}$.

\section{RESULTS}

Fetal body weights were $4.63 \pm 1.02 \mathrm{~kg}$ (range 2.88 to $5.90 \mathrm{~kg}$ ). During the control period, arterial $\mathrm{pH}, \mathrm{Po}_{2}$, $\mathrm{PCO}_{2}$, and $\mathrm{O}_{2}$ saturation were within the normal range for fetuses at this stage of gestation (Table 1 ). As expected, arterial $\mathrm{Po}_{2}$ and $\mathrm{O}_{2}$ saturation increased with oxygen ventilation. There was a decrease in $\mathrm{PCO}_{2}$ and an increase in $\mathrm{pH}$ due to mild hyperventilation of the fetuses. Occlusion of the umbilical cord did not cause any further alterations in fetal arterial blood gases or $\mathrm{pH}$.

Hemodynamic measurements taken at each stage of the experiment are summarized in Table 2. Ventilation caused a significant increase in LV output, principally as a result of increased stroke volume; heart rate remained relatively stable. The effects of ventilation on fetal PV loops are presented in Figure 3. LV end-diastolic volume and ejection fraction increased with ventilation. Although systolic blood pressure remained unchanged, $\mathrm{E}_{\mathrm{aLv}}$ decreased. In utero ventilation was not associated with a change in either $\mathrm{dP} / \mathrm{dt}_{\max }$ or $\mathrm{E}_{\mathrm{es}}$. Measurements taken while the umbilical cord was being occluded showed a transient increase in end-systolic pressure (17.7

Table 1. Fetal arterial blood gases during experiments*

\begin{tabular}{lccc}
\hline & Control & $\mathrm{O}_{2}$ ventilation & Occlusion \\
\hline $\mathrm{pH}$ & $7.37 \pm 0.03$ & $7.46 \pm 0.06 \dagger$ & $7.45 \pm 0.06$ \\
$\mathrm{PO}_{2}(\mathrm{kPa})$ & $3.29 \pm 0.68$ & $17.88 \pm 12.18 \ddagger$ & $17.17 \pm 10.34$ \\
$\mathrm{PCO}_{2}(\mathrm{kPa})$ & $5.71 \pm 0.81$ & $4.21 \pm 0.56 \dagger$ & $3.85 \pm 0.80$ \\
$\mathrm{O}_{2}$ saturation $(\%)$ & $46.2 \pm 9.7$ & $95.3 \pm 5.8 \dagger$ & $96.6 \pm 3.5$ \\
\hline
\end{tabular}

* Data are presented as mean $\pm 1 \mathrm{SD}$. Occlusion data were obtained 20 min after umbilical cord occlusion.

$\dagger p<0.001 v s$ the value during the immediately preceding stage.

$\ddagger p<0.05$ vs the value during the immediately preceding stage.

Table 2. Fetal hemodynamic measurements during experiments*

\begin{tabular}{lccc}
\hline & Control & $\mathrm{O}_{2}$ ventilation & Occlusion \\
\hline Heart rate (beats/min) & $153 \pm 15$ & $164 \pm 20$ & $158 \pm 26$ \\
EDV (mL/kg) & $2.3 \pm 0.9$ & $2.9 \pm 0.6 \dagger$ & $3.0 \pm 0.7$ \\
Stroke volume $(\mathrm{mL} / \mathrm{kg})$ & $1.2 \pm 0.3$ & $1.9 \pm 0.2 \ddagger$ & $1.7 \pm 0.2$ \\
Ejection fraction $(\%)$ & $52.8 \pm 11.1$ & $66.4 \pm 8.9 \ddagger$ & $61.2 \pm 12.5$ \\
$\mathrm{LV}$ output $(\mathrm{mL} / \mathrm{kg} / \mathrm{min})$ & $179.7 \pm 51.9$ & $311.6 \pm 59.6 \S$ & $280.7 \pm 77.3$ \\
$\mathrm{E}_{\mathrm{aLV}}(\mathrm{kPa} / \mathrm{mL})$ & $1.80 \pm 0.37$ & $1.04 \pm 0.33 \S$ & $1.09 \pm 0.47$ \\
$\mathrm{E}_{\mathrm{es}}(\mathrm{kPa} / \mathrm{mL})$ & $1.57 \pm 0.37$ & $1.41 \pm 0.47$ & $1.49 \pm 0.43$ \\
$\mathrm{dP} / \mathrm{dt}_{\max }(\mathrm{kPa} / \mathrm{s})$ & $201 \pm 30$ & $229 \pm 53$ & $237 \pm 47$ \\
Systolic pressure $(\mathrm{kPa})$ & $9.41 \pm 0.33$ & $9.33 \pm 0.75$ & $9.36 \pm 0.68$ \\
\hline
\end{tabular}

* Data are presented as mean $\pm 1 \mathrm{SD}$. Occlusion data were obtained 20 min after umbilical cord occlusion. EDV, end-diastolic volume. $\dagger p<0.05 v s$ the value during the immediately preceding stage.

$\ddagger p<0.001 v s$ the value during the immediately preceding stage.

$\S p<0.01 v s$ the value during the immediately preceding stage.

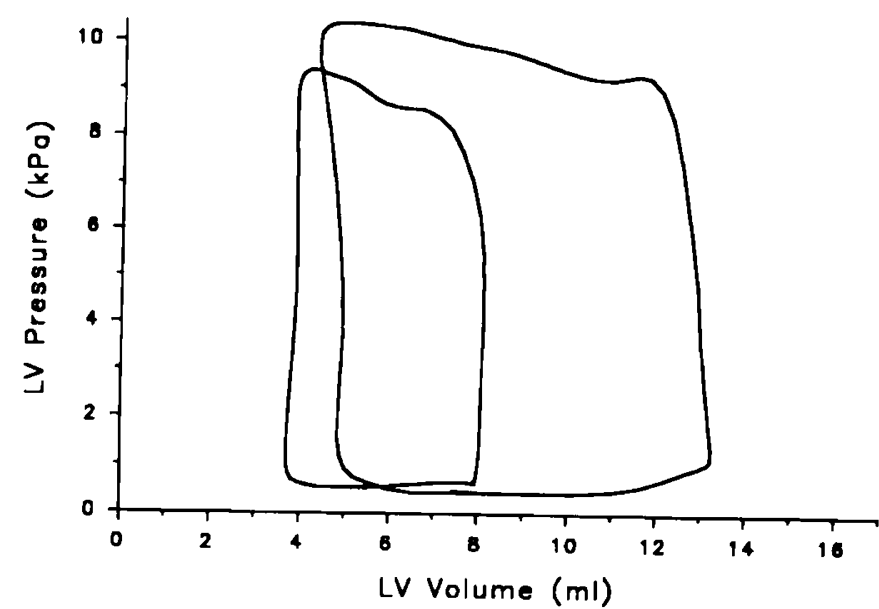

Figure 3. Left-ventricular PV loops recorded at control (smaller loop) and during oxygen ventilation.

$\pm 8.5 \%, p<0.001)$ and a decrease in stroke volume $(11.2$ $\pm 6.3 \%, p<0.01)$, reflecting an increase in $\mathrm{E}_{\mathrm{aLV}}(33.5 \pm$ $16.2 \%, p<0.01$ ) (Fig. 4). Twenty min after cord occlusion, however, all hemodynamic parameters returned to levels not significantly different from those obtained with ventilation alone (Table 2).

With ventilation alone, the increase in end-diastolic volume resulted in $58.4 \pm 20.5 \%$ and the reduction in $E_{a L V}$ caused $45.4 \pm 4.0 \%$ of the observed increase in stroke volume. After cord occlusion, the relative contributions to the increase in stroke volume from baseline were $60.2 \pm 3.7 \%$ and $46.6 \pm 8.9 \%$, respectively. The difference between these contributions did not reach statistical significance at either stage of the experiment.

\section{DISCUSSION}

Using the conductance catheter technique, absolute fetal LV volumes were measured during in utero ventilation. We found a marked increase in LV end-diastolic volume, stroke volume, and ejection fraction associated

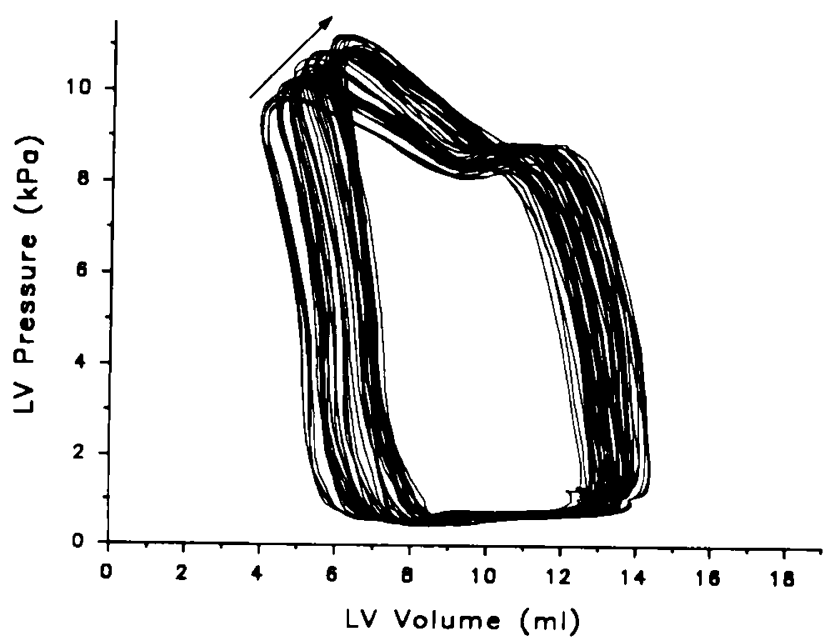

Figure 4. PV loops acquired during occlusion of the umbilical cord showing a definite increase in afterload. The arrow indicates the direction of change during umbilical occlusion. 
with a reduction in effective $L V$ afterload as measured by $E_{a L v}$. The increased end-diastolic volume further augmented stroke volume, allowing systolic blood pressure to be maintained after the transition. Contractility, as assessed by $E_{e s}$, was found to play no role in the increased LV output observed with in utero ventilation.

This experimental protocol was used to study the mechanism by which the fetal LV dramatically increases its output at birth. Previous studies showed that in utero oxygen ventilation creates a central circulatory pattern identical with that in the immediate postnatal period (18, $19,32,33)$. The arterial blood gases obtained at a time when the umbilical cord was occluded support the notion that the lungs became the sole site for gas exchange. For this to occur, effective ventilation must have been linked to a significant reduction in pulmonary resistance and increased flow.

As previously shown $(18,19)$, we also found a marked increase in LV output during in utero ventilation. Heart rate changes, which have been suggested to contribute to this increase (34), were not found; rather, we observed a profound increase in LV stroke volume similar to that reported in other studies $(18,19)$. Associations between the determinants of LV output, i.e. contractility, preload, and afterload, and the increase in stroke volume have previously been speculated on.

In this study, contractility as assessed by $E_{e s}$ was found not to change at a time when LV output almost doubled. The conventional method of determining $E_{e s}$ requires reduction of $L V$ preload, which is usually accomplished by occlusion of the inferior vena cava (35). Application of this technique in the fetus has previously been found to yield inconsistent results (24); the reasons for this were speculated to be related to the unique ventricular interaction and right ventricular dominance of the fetal heart. Because of the difficulties associated with measuring $E_{e s}$ using caval occlusions, a single-beat method of estimating $E_{e s}$ was used. Although the singlebeat $E_{e s}$ has not yet been fully validated, the assumption that contractility did not change is supported by the fact that $\mathrm{dP} / \mathrm{dt}_{\max }$ also remained unchanged. The lack of change in $\mathrm{dP} / \mathrm{dt}_{\max }$ despite the marked increase in enddiastolic volume could be interpreted as a decrease in contractility. However, we do not expect this to be the case, because the added demands on the LV with transition would tend to elicit a positive contractile response. We suggest that $\mathrm{dP} / \mathrm{dt}_{\max }$ remained stable more as a result of rightward septal displacement than of free wall distension, a phenomenon observed in human fetuses and infants (36). Therefore, the increase in end-diastolic volume may not have been associated with a comparable degree of free-wall fiber stretching or preload.

Other studies suggested that contractility, as assessed by $\mathrm{dP} / \mathrm{dt}_{\max }$, fractional shortening, and postextrasystolic potentiation, in lambs studied 18-24 h before delivery and $2-3 \mathrm{~h}$ after birth increased significantly (16). Similar findings were reported in the fetal and postnatal lamb heart in intact animals as well as in isolated cardiac muscle (17).
Those studies, however, did not examine changes in contractility immediately surrounding the transition. Furthermore, the absence of changes in heart rate during the same period and the previous observation that the transition still occurs in the presence of $\beta$-adrenergic blockade (18) imply that no significant sympathetic-driven changes in contractility occur. Although it may be true that substantial changes in contractility do occur during the perinatal period, these do not appear to be essential for the immediate circulatory adjustments at birth.

The marked increase in end-diastolic volume observed in this study may have involved diminution of right ventricular constraint on LV filling. The fetal heart is characterized by RV dominance, with filling pressures equal to or greater than those of the LV (1-3). The LV of the fetus is more ellipsoidal than that of the neonatal child as a result of leftward septal buckling (36). The reduction in RV filling pressure associated with ventilation (32) could result in a change in septal alignment, causing an increase in LV chamber compliance and thus augmenting LV filling. It has also recently been suggested that pressures exerted by tissues surrounding the fetal heart significantly restrict LV filling (37) and that ventilation reduces this constraint (38).

The increase in LV stroke volume observed with ventilation is more than can be ascribed to additional volume loading, because the fetal LV is known to be functioning near the plateau of its Frank-Starling curve (39), although when afterload is controlled LV output can increase in response to increased filling pressures (40). Rather than moving along its cardiac function curve, it is more likely that the ventricle operates on a higher curve, as previously described (18). The significant decrease in $E_{a L V}$, in addition to a possible increase in LV chamber compliance, might explain such a shift (Fig. 5).

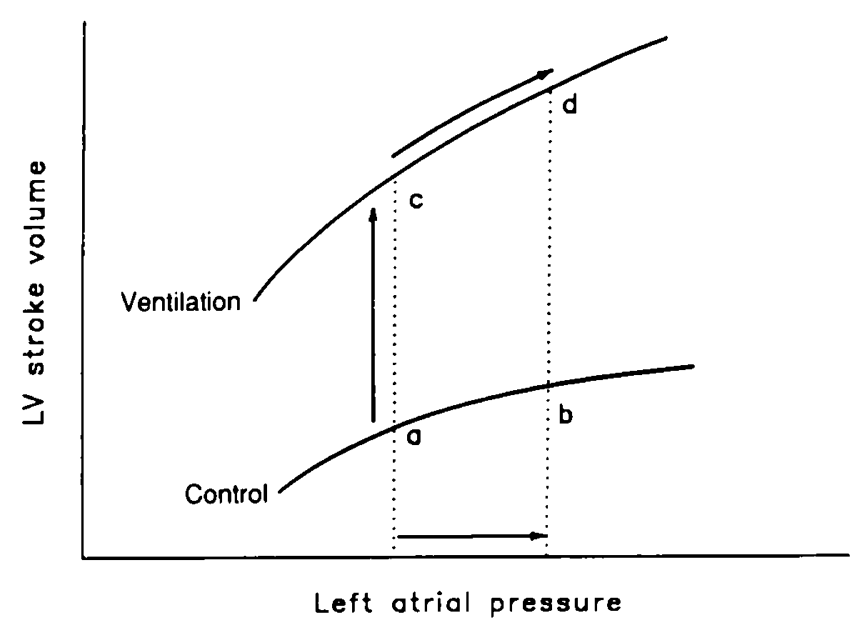

Figure 5. Diagram of LV function curves. In the fetus, the LV is known to operate near the plateau of the curve $(a)$, such that increases in atrial pressure $(b)$ result in marginal increases in stroke volume. During oxygen ventilation, the decreased afterload and increased compliance cause the LV to operate on an elevated curve $(c)$. Because the new curve is steeper, it is possible that the LV gains preload reserve in the process $(d)$. 
Afterload, expressed as arterial elastance, relates stroke volume to the resulting developed pressure (22, 23). In the fetus, both ventricles eject in parallel into the same arterial system; thus, afterload is a function of the combined stroke volumes and the resulting developed pressure. We hypothesize that the effective afterload that each ventricle encounters is, in part, governed by the volume of blood ejected by the opposite ventricle. At the transition, because the RV no longer contributes to aortic flow, the arterial tree has a larger capacity to be filled by the LV. Thus, the LV is, in effect, exposed to a lower afterload, allowing stroke volume to increase. A further decrease in afterload may be explained by the exposure of the LV to the lower-resistance pulmonary circulation through the ductus arteriosus. A left-to-right shunt through the ductus, accounting for up to $30 \%$ of $\mathrm{LV}$ output, has been found to exist in the early transitional circulation $(11,41,42)$.

The relative contributions of the increase in enddiastolic volume and the decrease in $E_{a L V}$ to augmentation of stroke volume were estimated using PV analysis and were found to contribute equally. The combined effects accounted for the total observed increase in stroke volume, corroborating the finding that contractility did not change. The impact of end-diastolic volume and $E_{a L v}$ on stroke volume did not change after umbilical cord occlusion.

On occlusion of the umbilical cord, afterload increased significantly; however, this response was transient, and after $20 \mathrm{~min}$ all hemodynamic effects of the occlusion disappeared. This indicates the possible existence of adaptive vasodilator mechanisms that may also play a role in regulating afterload during transition.

To our knowledge, these are the first reported ejection fractions in the fetus based on absolute volume determinations. Although measurement of absolute LV volume using the conductance catheter may be accurate, the technique has yet to be validated in the fetus. Previous studies using endocardial ultrasonic crystals to measure LV dimensions in fetal sheep reported a fractional shortening of $24.3 \%(43,44)$. Assuming a circular cross section cylindrical geometry, the resulting segmental ejection fraction would have been about $43 \%$, compared with $52.8 \%$ calculated in this study. Left ventricular ejection fraction increased with in utero ventilation and, because $E_{e s}$ was found not to change, this increase must have been due to the reduction in afterload.

All of the increase in LV output in this study occurred with oxygen ventilation alone; occlusion of the umbilical cord caused no further augmentation. This finding is in agreement with previous observations (19) and is also supported by reports that almost the entire decrease in pulmonary vascular resistance, cessation of foramen ovale flow, and most of the ductus arteriosus left-to-right shunt occur with oxygen ventilation before occlusion of the umbilical cord $(19,33)$.

In summary, this study helps to elucidate the mechanisms by which the fetal LV increases its output at birth.
PV analysis revealed that increased end-diastolic volume and reduced effective $L V$ afterload are the major determinants of, and contribute to a comparable extent to, the augmented LV stroke volume. This increase occurs in the absence of any apparent change in LV contractility.

Acknowledgment. The authors thank Charlene Small for her excellent technical assistance.

\section{REFERENCES}

1. Anderson DF, Bissonette JM, Faber JJ, Thornburg KL 1981 Central shunt flows and pressures in the mature fetal lamb. Am J Physiol 241:H60-H66

2. Heymann MA, Creasy RK, Rudolph AM 1973 Quantitation of blood flow pattern in the foetal lamb in utero. In: Foetal and Neonatal Physiology, Proceedings of the Sir Joseph Bancroft Centenary Symposium. Cambridge University Press, Cambridge, UK, pp 129-135

3. Pinson CW, Morton MJ, Thornburg KL 1987 An anatomic basis for fetal right ventricular dominance and arterial pressure sensitivity. J Dev Physiol 9:253269

4. Berman Jr W, Musselman J 1979 Myocardial performance in the newborn lamb. Am J Physiol 237:H66-H70

5. Klopfenstein HS, Rudolph AM 1978 Postnatal changes in the circulation and responses to volume loading in sheep. Circ Res 42:839-845

6. Lister G, Walter TK, Versmold HT, Dallman PR, Rudolph AM 1979 Oxygen delivery in lambs: cardiovascular and hematologic development. Am J Physiol 237: $\mathrm{H} 668-\mathrm{H} 675$

7. Minoura S, Gilbert RD 1987 Postnatal change in cardiac function in lambs: effects of ganglionic block and afterload. J Dev Physiol 9:123-135

8. Riemenschneider TA, Brenner RA, Mason DA 1981 Maturational changes in myocardial contractile state of newborn lambs. Pediatr Res 15:349-356

9. Romero TE, Friedman WF 1979 Limited left ventricular response to volume overload in the neonatal period: a comparative study with the adult animal. Pediatr Res 13:910-915

10. Teitel DF, Sidi D, Chin T, Brett C, Heymann MA, Rudolph AM 1985 Developmental changes in myocardial contractile reserve in the lamb. Pediatr Res 19:948-955

11. Assali NS, Morris JA, Beck R 1965 Cardiovascular hemodynamics in the fetal lamb before and after lung expansion. Am J Physiol 208:122-129

12. Dawes GS, Mott JC, Widdicombe JG, Wyatt DG 1953 Changes in the lungs of the newborn lamb. J Physiol 121:141-162

13. Dawes GS 1968 The pulmonary circulation in the foetus and newborn. In: Foetal and Neonatal Physiology. Yearbook Medical Publishers, Chicago, pp 79-9)

14. Heymann MA, Rudolph AM, Nies AS, Melmon KL 1969 Bradykinin production associated with oxygenation of the fetal lamb. Circ Res 25:521-534

15. Cassin S, Dawes GS, Mott JC, Ross BB, Strang LB 1964 The vascular resistance of the foetal and newly ventilated lung of the lamb. J Physiol $171: 61-79$

16. Anderson PAW, Manring A, Glick KL, Crenshaw CC 1982 Biophysics of the developing heart. III. A comparison of the left ventricular dynamics of the fetal and neonatal lamb heart. Am J Obstet Gynecol 143:195-203

17. Anderson PAW, Glick KL, Manring A, Crenshaw CC 1984 Developmental changes in cardiac contractility in fetal and postnatal sheep: in vitro and in vivo. Am J Physiol 247:H371-H379

18. Morton MJ, Pinson CW. Thornburg KL 1987 In utero ventilation with oxygen augments left ventricular stroke volume in lambs. J Physiol 383:413-424

19. Teitel DF, Iwamoto HS, Rudolph AM 1987 Effects of birth-related events on central blood flow patterns. Pediatr Res 22:557-566

20. Kass D, Maughan WL, Mao Guo Z, Kono A, Sunagawa K, Sagawa K 1987 Comparative influence of load versus inotropic states on indexes of ventricular contractility: experimental and theoretical analysis based on pressurevolume relationships. Circulation 76:1422-1436

21. Sagawa K, Maughan WL, Suga H, Sunagawa K 1988 In: Cardiac Contraction and the Pressure-Volume Relationship. Oxford University Press, New York, pp $111-119$

22. Kass $D$, Grayson R, Marino $P 1990$ Pressure-volume analysis as a method for quantifying simultaneous drug (Amrinone) effects on arterial load and contractile state in vivo. J Am Coll Cardiol 16:726-732

23. Sunagawa K, Maughan WL, Burkhoff D, Sagawa K 1983 Left ventricular interaction with arterial load studied in isolated canine ventricle. Am J Physiol
245: $7773-\mathrm{H} 780$

24. Lewinsky RM, Szwarc RS, Benson LN, Ritchie JWK 1993 The effects of hypoxic acidemia on left ventricular end-systolic elastance in fetal sheep. Pediatr Res 34:38-43

25. Baan J, Aouw Jong TT, Kerkhof PLM, Moene RJ, van Dijk AD, van der Velde ET, Koops J 1981 Continuous stroke volume and cardiac output from intraventricular dimensions obtained with impedance catheter. Cardiovasc Res 15:328-334

26. Baan J, van der Velde ET, de Bruin HG, Smeenk GJ, Koops J, van Dijk AD, Temmerman D, Senden J, Buis B 1984 Continuous measurement of left 
ventricular volume in animals and humans by conductance catheter. Circulation $70: 812-823$

27. Burkhoff D, van der Velde ET, Kass D, Baan J, Maughan WL, Sagawa K 1985 Accuracy of volume measurement by conductance catheter in isolated, ejecting canine hearts. Circulation 72:440-447

28. Cassidy SC, Teitel DF 1990 The conductance catheter technique for measurement of left ventricular volume in young piglets. Pediatr Res 27:16A(abstr)

29. Kass D, Yamazaki T, Burkhoff D, Maughan WL, Sagawa K 1986 Determination of left ventricular end-systolic pressure-volume relationships by the conductance (volume) catheter technique. Circulation 73:586-595

30. Teitel DF, Klautz R, Steendijk P, van der Velde ET, van Bel F, Baan J 1991 The end-systolic pressure-volume relationship in the newborn lamb: effects of loading and inotropic interventions. Pediatr Res 29:473-482

31. Szwarc RS, Cleveland D, Benson LN 1992 Left-ventricular isovolumic de veloped pressure $\left(P_{\max }\right)$ estimated from single ejecting contractions. Mol Cell Cardiol 24(suppl V):37(abstr)

32. Reller MD, Morton MJ, Reid DL. Thornburg KL 1987 Fetal lamb ventricles respond differently to filling and arterial pressures and to in utero ventilation. Pediatr Res 22:621-626

33. Teitel DF, Iwamoto HS, Rudolph AM 1990 Changes in the pulmonary circulation during birth-related events. Pediatr Res 27:372-378

34. Anderson PAW, Glick KL, Killam AP, Mainwaring RD 1986 The effect of heart rate on in utero left ventricular output in the fetal sheep. J Physiol $372: 557-573$
35. Baan J, van der Velde ET 1988 Sensitivity of left ventricular end-systolic pressure-volume relationships to type of loading intervention in dogs. Circ Res 62:1247-1258

36. Azancot A, Caudell TP, Allen HD, Horowitz S, Sahn DJ, Stoll C, Thies C, Valdes-Cruz LM, Goldberg SJ 1983 Analysis of ventricular shape by echocardiography in normal fetuses, newborns, and infants. Circulation 68:1201-1211

37. Grant DA, Maloney JE, Tyberg JV, Walker AM 1992 Effects of external constraint on the fetal left ventricular function curve. Am Heart J 123:16011609

38. Grant DA, Condo CS, Maloney JE, Walker AM, Tyberg JV 1992 Changes in pericardial pressure during the perinatal period. Circulation $86: 1615-1621$

39. Gilbert RD 1980 Control of fetal cardiac output during changes in blood volume. Am J Physiol 238:H80-H86

40. Hawkins J, Van HG, Schmidt KG, Rudolph AM 1989 Effects of increasing afterload on left ventricular output in fetal lambs. Circ Res 65:127-134

41. Assali NS, Sehgal N, Marable S 1962 Pulmonary and ductus arteriosus circulation in the fetal lamb before and after birth. Am J Physiol 202:536-540

42. Dawes GS, Mott JC, Widdicombe JG 1955 Closure of the foramen ovale in newborn lambs. J Physiol 128:384-395

43. Kirkpatrick SE, Covell JW, Friedman WF 1973 A new technique for the continuous assessment of fetal and neonatal cardiac performance. Am J Obstet Gynecol 116:963-972

44. Kirkpatrick SE, Pitlick PT, Naliboff J, Friedman WF 1976 Frank-Starling relationship as an important determinant of fetal cardiac output. Am J Physiol 231:495-500 